\title{
SUPPORT POINTS AND DOUBLE POLES
}

\author{
SAY SONG GOH
}

(Communicated by Albert Baernstein II)

\begin{abstract}
This paper gives some sufficient conditions for support points of the class $S$ of univalent functions to be rotations of the Koebe function $k(z)=$ $z(1-z)^{-2}$. If $f$ is a support point associated with a continuous linear functional $L$ and if the function $\Phi(w)=L\left(f^{2} /(f-w)\right)$ does not have a double pole, then under some mild additional assumptions, a rational support point $f$ must be a rotation of the Koebe function. The situation is more complicated when $\Phi$ has a double pole. However, we are able to prove the two-functional conjecture for derivative functionals, where $\Phi$ has a double pole.
\end{abstract}

\section{INTRODUCTION}

Let $S$ be the class of functions $f$ analytic and univalent in the unit disk $\mathbb{D}$ such that $f(0)=0$ and $f^{\prime}(0)=1$. The leading example is the Koebe function $k(z)=z(1-z)^{-2}$, which maps the unit disk onto the entire complex plane minus the part of the negative real axis from $-\frac{1}{4}$ to infinity. Let $\mathscr{H}(\mathbb{D})$ be the space of all analytic functions in $\mathbb{D}$, with the usual topology of uniform convergence on compact subsets. A function $f \in S$ is called a support point of $S$ if there is a continuous linear functional $L$ on $\mathscr{H}(\mathbb{D})$, nonconstant on $S$, such that $f$ maximizes $\operatorname{Re}\{L\}$ over $S$. It is well known (see [2, pp. 286$288,306-307])$ that a support point of $S$ maps $\mathbb{D}$ onto the complement of a single analytic arc extending to infinity which satisfies the differential equation $L\left(f^{2} /(f-w)\right)(d w / w)^{2}>0$. The Koebe function and its rotations are support points of $S$, but there are many more.

In this paper we obtain some sufficient conditions for support points to be rotations of the Koebe function. Much of our discussion will be centered around the two-functional conjecture, which says that if a function $f \in S$ maximizes $\operatorname{Re}\{L\}$ are $\operatorname{Re}\{M\}$ for two continuous linear functionals $L$ and $M$ which are linearly independent and nonconstant on $S$, then $f$ has to be a rotation of the Koebe function. (A more complete study of the two-functional conjecture can be found in [3].)

If $f$ is a support point associated with a functional $L$ such that the function $\Phi(w)=L\left(f^{2} /(f-w)\right)$ has no double poles and if some additional conditions

Received by the editors May 26, 1992 and, in revised form, January 5, 1993.

1991 Mathematics Subject Classification. Primary 30C50; Secondary 30C70.

Key words and phrases. Univalent functions, variational methods, algebraic functions, the twofunctional conjecture, derivative functionals. 
are satisfied, then we show in $\S 2$ that $f$ is a rotation of the Koebe function. Here a double pole means a pole of order two. In $\S 3$ we prove the two-functional conjecture for derivative functionals, where $\Phi$ has a double pole.

\section{RATIONAL SUPPORT POINTS}

In 1950 Schaeffer and Spencer [6] proved the two-functional conjecture for a special class of coefficient functionals. In [3] we adapted their method to prove the two-functional conjecture for several other cases. An important step in the method of Schaeffer and Spencer is to show that a certain rational support point is a rotation of the Koebe function. In this connection the following theorem of Srebro [7] is often useful.

Theorem (Srebro). Suppose that $f \in S$ is a rational function which maps the unit disk onto the complement of an arc $\Gamma$ extending to infinity. Then $\Gamma$ is a half-line, and $f$ is a Marty transform of the Koebe function.

By adding some assumptions involving double poles, we shall improve this result to provide a sufficient condition for a rational support point to be a rotation of the Koebe function.

Remarks. (i) Although the above theorem was proven independently by Srebro, it turned out that the result was known much earlier. Proofs had been given by L. Fuchs in 1874 (J. Reine Angew. Math. 77, 339-352), R. Stolzenberg in 1928 (Jber. Deutsch. Math.-Verein. 37, 64-68), and L. Bieberbach in 1940 (Viert. Natursfor. Ges. Zürich 85, 143-148). However, Srebro's proof is simpler than the previous ones.

(ii) A function $f \in S$ is said to be a Marty transform of the Koebe function if $f=\phi \circ k \circ \psi$, where $k$ is the Koebe function and $\phi$ and $\psi$ are Möbius transformations. As noted by Srebro [7], this is equivalent to

$$
f(z)=A\left[\left(\frac{z-a}{z-b}\right)^{2}-\left(\frac{a}{b}\right)^{2}\right]
$$

for some distinct points $a$ and $b$ on the unit circle and $A=b^{3} /[2 a(a-b)]$. A simple manipulation leads to

$$
f(z)=A \frac{\left[1-(a / b)^{2}\right] z^{2}+2\left[\left(a^{2} / b\right)-a\right] z}{(z-b)^{2}} .
$$

Geometrically, a function in $S$ is a Marty transform of the Koebe function if and only if it maps the unit disk onto the complement of a half-line, which need not be radial.

We shall need only the following direct consequence of Srebro's theorem here.

Corollary. If $f \in S$ is a rational support point, then $f$ is given by (1).

Remark. If $1-(a / b)^{2}=0$, then an easy calculation shows that the function $f$ given by (1) is simply a rotation of the Koebe function. We shall use this observation to prove the following theorem.

Theorem 1. Let $f \in S$ be a rational support point associated with a continuous linear functional $L$ of rational type. Define

$$
\Phi(w)=L\left(f^{2} /(f-w)\right) .
$$


Suppose that $\Phi(0) \neq 0, \infty$. If $\Phi$ has no double poles, then $f$ is a rotation of the Koebe function.

A functional $L$ is said to be of rational type if $\Phi(w)=L\left(f^{2} /(f-w)\right)$ is a rational function for each $f \in S$. (See Brickman [1].)

Proof of Theorem 1. Let $z_{0}$ be the point on the unit circle at which $f\left(z_{0}\right)=w_{0}$, where $w_{0}$ is the finite tip of the omitted arc $\Gamma$. Since $f \in S$ maximizes $\operatorname{Re}\{L\}$, it follows that $\Gamma$ satisfies

$$
\Phi(w)(d w / w)^{2}>0
$$

where $\Phi$ is defined by (2).

It is known (see [2, pp. 312-313]) that if $\Phi\left(w_{0}\right)=0$, then $f$ is a rotation of the Koebe function. Thus we may assume that $\Phi\left(w_{0}\right) \neq 0$. By the usual method of Schwarz reflection beyond $\mathbb{D},(3)$ implies that $w=f(z)$ satisfies

$$
\Phi(w)\left(z w^{\prime} / w\right)^{2}=\mathscr{R}(z),
$$

where $\mathscr{R}$ is a rational function in $z$ since $\Phi$ is a rational function in $w$. Apart from a double zero at $z_{0}, \mathscr{R}$ is real, positive and finite on the unit circle. (Note that $\Phi$ has a simple zero at infinity because it is known that $L\left(f^{2}\right) \neq 0$. See [2, pp. 307-310].) Observe also that $z w^{\prime} / w$ has neither zeros nor poles in $\mathbb{D}$. Hence, since $\Phi$ has no double poles, $\mathscr{R}$ has no double poles.

By the corollary to Srebro's theorem, the extremal function $f$ is given by the expression (1). As remarked before, it suffices to show that $1-(a / b)^{2}=0$. If $1-(a / b)^{2} \neq 0$, then $f$ has a simple zero at $z=\xi$ for some $|\xi| \geq 1$. As $z \rightarrow \xi$, $w=f(z) \rightarrow 0$ and $\Phi(w) \rightarrow \Phi(0)$. By hypothesis, $\Phi(0) \neq 0, \infty$. Thus, in a neighborhood of $z=\xi$, the left-hand side of $(4)$ has the form $b(z-\xi)^{-2}+\cdots$ for some constant $b$. This is a contradiction since $\mathscr{R}$ has no double poles, and the proof is complete.

Remarks. (i) The hypothesis of $\Phi$ having no double poles is essential. Pearce [5] showed that many Marty transforms of the Koebe function, not rotations, are support points associated with derivative functionals $L(f)=e^{-i \sigma} f^{\prime}(\zeta)$ $(0 \neq \zeta \in \mathbb{D}, \sigma \in \mathbb{R}) ;$ for such functionals, $\Phi$ defined by (2) has a double pole. (See §3.)

(ii) It is immediate from Theorem 1 that all rational support points associated with point-evaluation functionals $L(f)=e^{-i \sigma} f(\zeta) \quad(0 \neq \zeta \in \mathbb{D}, \sigma \in \mathbb{R})$ are rotations of the Koebe function. Theorem 1 can also be used to show (see [4]) that all rational support points associated with functionals of the form

$$
L(f)=\sum_{k=1}^{K} \sum_{n=2}^{N_{k}} a_{n k} f^{(n)}\left(\zeta_{k}\right)
$$

$\left(N_{k} \geq 2,0 \neq \zeta_{k} \in \mathbb{D}\right.$ for $1 \leq k \leq K$ and $a_{n k} \in \mathbb{C}$ for $\left.1 \leq k \leq K, 2 \leq n \leq N_{k}\right)$ are rotations of the Koebe function.

(iii) If the function $\Phi$ given by (2) has either a pole or a zero at the origin, we can easily modify the proof of Theorem 1 to produce analogous results. Thus $f$ is a rotation of the Koebe function if

(a) $\Phi$ has a pole of order $m(m \geq 1)$ at $w=0$ and no poles of order $(m+2)$ elsewhere; 
(b) $\Phi$ has a zero of order $m(m \geq 3)$ at $w=0$ and no zeros of order $(m-2)$ elsewhere; or

(c) $\Phi$ has a simple zero at $w=0$ and no simple poles elsewhere.

However, when $\Phi$ has a double zero at $w=0$, the idea used in proving Theorem 1 is inconclusive. Also, the above sufficient conditions may not be the best possible.

\section{PRoof of THE TWO-FUNCTIONAL CONJECTURE FOR DERIVATIVE FUNCTIONALS}

In this section we shall prove the two-functional conjecture for functionals of the form $L(f)=e^{-i \sigma} f^{\prime}(\zeta)$, where $0 \neq \zeta \in \mathbb{D}$ and $\sigma \in \mathbb{R}$. Note that here the function $\Phi(w)=L\left(f^{2} /(f-w)\right)$ has a double pole.

Theorem 2. Suppose that $f \in S$ maximizes $\operatorname{Re}\{L\}$ and $\operatorname{Re}\{M\}$, where

$$
L(f)=\left(e^{-i \sigma_{1}}\right) f^{\prime}\left(\zeta_{1}\right) \text { and } M(f)=\left(e^{-i \sigma_{2}}\right) f^{\prime}\left(\zeta_{2}\right),
$$

with $0 \neq \zeta_{1}, \zeta_{2} \in \mathbb{D}, \zeta_{1} \neq \zeta_{2}$, and $\sigma_{1}, \sigma_{2} \in \mathbb{R}$. Then $f$ is a rotation of the Koebe function.

Remark. The assumption $\zeta_{1} \neq \zeta_{2}$ is equivalent to the functionals $L$ and $M$ being linearly independent. Indeed, if $L=c M$ for some $c \in \mathbb{C}$, then for all functions $g$ in the class $S,\left(e^{-i \sigma_{1}}\right) g^{\prime}\left(\zeta_{1}\right)=c\left(e^{-i \sigma_{2}}\right) g^{\prime}\left(\zeta_{2}\right)$. By considering first the identity $g_{1}(z)=z$ and then the function $g_{2}(z)=z+z^{2} / 2$ in $S$, we obtain $\zeta_{1}=\zeta_{2}$.

Proof of Theorem 2. Since $f \in S$ maximizes $\operatorname{Re}\{L\}$, the omitted arc $\Gamma$ satisfies the differential equation $L\left(f^{2} /(f-w)\right)(d w / w)^{2}>0$, giving

$$
\frac{-2 e^{-i \sigma_{1}}\left(B_{1} C_{1}\right)\left(w-B_{1} / 2\right)}{\left(w-B_{1}\right)^{2}}\left(\frac{d w}{w}\right)^{2}>0,
$$

where $B_{1}=f\left(\zeta_{1}\right)$ and $C_{1}=f^{\prime}\left(\zeta_{1}\right)$.

Let $z_{0}$ be the point on the unit circle such that $f\left(z_{0}\right)=w_{0}$, where $w_{0}$ is the finite tip of $\Gamma$. As in the proof of Theorem 1, we may assume that $\Phi(w)=L\left(f^{2} /(f-w)\right)$ does not vanish at $w_{0}$. Then $B_{1} / 2 \notin \Gamma$, so there exists $0 \neq z_{1} \in \mathbb{D}$ such that $f\left(z_{1}\right)=B_{1} / 2$. Clearly, $z_{1} \neq \zeta_{1}$. By the method of Schwarz reflection beyond $\mathbb{D},(5)$ implies that

$$
\frac{\left(w-B_{1} / 2\right)}{\left(w-B_{1}\right)^{2}}\left(\frac{z w^{\prime}}{w}\right)^{2}=\frac{A_{1}\left(z-z_{0}\right)^{2}\left(z-z_{1}\right)\left(z-1 / \bar{z}_{1}\right)}{\left(z-\zeta_{1}\right)^{2}\left(z-1 / \bar{\zeta}_{1}\right)^{2}},
$$

where $w=f(z)$ and $A_{1}$ is some constant.

Similarly, since $f \in S$ maximizes $\operatorname{Re}\{M\}$, we obtain

$$
\frac{\left(w-B_{2} / 2\right)}{\left(w-B_{2}\right)^{2}}\left(\frac{z w^{\prime}}{w}\right)^{2}=\frac{A_{2}\left(z-z_{0}\right)^{2}\left(z-z_{2}\right)\left(z-1 / \bar{z}_{2}\right)}{\left(z-\zeta_{2}\right)^{2}\left(z-1 / \bar{\zeta}_{2}\right)^{2}},
$$

where $w=f(z), 0 \neq z_{2} \in \mathbb{D}, f\left(z_{2}\right)=B_{2} / 2, z_{2} \neq \zeta_{2}$, and $A_{2}$ is some constant.

Observe that $z_{1} \neq z_{2}$. Indeed, if $z_{1}=z_{2}$, then $f\left(z_{1}\right)=f\left(z_{2}\right)$, giving $B_{1}=B_{2}$. Since $f$ is univalent, it follows that $\zeta_{1}=\zeta_{2}$, a contradiction. 
We shall now adapt the method of Schaeffer and Spencer. Through division, the two differential equations (6) and (7) generate a common analytic continuation $w=F(z)$ of the given function $f \in S$ beyond the unit disk. This continuation $F$ is an algebraic function. It is analytic in the extended complex plane with the exception of a finite number of singularities which are either poles or algebraic branch points.

Let us first show that $F\left(z_{1}\right)=B_{1} / 2$ and $F\left(z_{2}\right)=B_{2} / 2$ on every sheet of the continuation. Suppose, on the contrary, that $F\left(z_{1}\right) \neq B_{1} / 2$ for some continuation. Then this continuation must have one of the local structures

$$
\begin{gathered}
w=F(z)=c\left(z-z_{1}\right)^{\gamma}+\cdots, \\
w=F(z)=c\left(z-z_{1}\right)^{-\gamma}+\cdots,
\end{gathered}
$$

or

$$
w=F(z)=\omega+c\left(z-z_{1}\right)^{\gamma}+\cdots, \quad \omega \neq 0, B_{1} / 2,
$$

near the point $z_{1}$, where $c \in \mathbb{C}, c \neq 0$, and $\gamma>0$. For the form (8), the left-hand side of (6) becomes $b\left(z-z_{1}\right)^{-2}+\cdots$ for some constant $b$. This contradicts the right-hand side of (6). For the form (9), the left-hand side of (6) becomes $b\left(z-z_{1}\right)^{\gamma-2}+\cdots$ for some constant $b$. Comparing this to the right-hand side of (6), we conclude that $\gamma=3$. This is impossible because (7) now implies that $z_{1}=z_{2}$, a contradiction. For the form (10), using (6), we obtain $\omega \neq B_{1}$ and $\gamma=\frac{3}{2}$. Now consider (7) with $\gamma=\frac{3}{2}$. Then it follows from the observation of $z_{1} \neq z_{2}$ that $\omega$ equals $B_{2} / 2$ or $B_{2}$. It is easily seen that $\omega=B_{2} / 2$ is impossible. But if $\omega=B_{2}$, then we are forced to have $\zeta_{2}=z_{1}$, which implies that $B_{2}=B_{1} / 2$, or $\omega=B_{1} / 2$. This is a contradiction, since the form (10) assumes $\omega \neq B_{1} / 2$. Hence all three forms (8)-(10) of $w=F(z)$ are impossible, and thus $F\left(z_{1}\right)=B_{1} / 2$ on every sheet of the continuation. Similarly, $F\left(z_{2}\right)=B_{2} / 2$ on every sheet of the continuation.

Next we show that $F$ is single-valued in $\mathbb{C}$. We shall consider the cases $B_{2} \neq B_{1} / 2$ and $B_{2}=B_{1} / 2$ separately. First suppose that $B_{2} \neq B_{1} / 2$. Dividing (6) by (7), we obtain

$$
\frac{\left(w-B_{1} / 2\right)\left(w-B_{2}\right)^{2}}{\left(w-B_{2} / 2\right)\left(w-B_{1}\right)^{2}}=\frac{A_{1}\left(z-z_{1}\right)\left(z-1 / \bar{z}_{1}\right)\left(z-\zeta_{2}\right)^{2}\left(z-1 / \bar{\zeta}_{2}\right)^{2}}{A_{2}\left(z-z_{2}\right)\left(z-1 / \bar{z}_{2}\right)\left(z-\zeta_{1}\right)^{2}\left(z-1 / \bar{\zeta}_{1}\right)^{2}},
$$

where $w=F(z)$. The left-hand side of (11) may be written as

$$
\frac{8\left(B_{1} / 2-B_{2}\right)^{2}}{B_{1}^{2}\left(B_{1}-B_{2}\right)}\left(w-B_{1} / 2\right)+\cdots,
$$

near the point $w=B_{1} / 2$. By assumption, $B_{1} / 2-B_{2} \neq 0$. Since $F\left(z_{1}\right)=B_{1} / 2$ on every sheet of the continuation, the inverse function theorem shows that (11) has a unique local solution $w=F(z)$ near $z_{1}$. Thus $F$ returns in every continuation to the same function element $f$ at $z_{1}$.

It now follows that $F$ is single-valued in the entire complex plane. Indeed, suppose that there exist two continuations from $z_{1}$ to a point $\eta$ along paths $C_{1}$ and $C_{2}$ which give different values $F(\eta)=\omega_{1}$ and $F(\eta)=\omega_{2}$. Then from the above we see that the continuation along the closed path $C_{1}-C_{2}$ returns $F$ to its original function element at $z_{1}$. Reversing the part of this continuation 
which extends over $-C_{2}$ now shows that the continuation along $C_{2}$ from $z_{1}$ to $\eta$ actually gives the value $F(\eta)=\omega_{1} \neq \omega_{2}$, a contradiction.

Next suppose that $B_{2}=B_{1} / 2$. Dividing (7) by (6), we obtain

$$
\frac{\left(w-B_{2} / 2\right)\left(w-B_{1}\right)^{2}}{\left(w-B_{1} / 2\right)\left(w-B_{2}\right)^{2}}=\frac{A_{2}\left(z-z_{2}\right)\left(z-1 / \bar{z}_{2}\right)\left(z-\zeta_{1}\right)^{2}\left(z-1 / \bar{\zeta}_{1}\right)^{2}}{A_{1}\left(z-z_{1}\right)\left(z-1 / \bar{z}_{1}\right)\left(z-\zeta_{2}\right)^{2}\left(z-1 / \bar{\zeta}_{2}\right)^{2}},
$$

where $w=F(z)$. We shall proceed as before, referring now to the point $B_{2} / 2$ and using the fact that $F\left(z_{2}\right)=B_{2} / 2$ for every continuation. The left-hand side of (12) can be expressed as

$$
\frac{8\left(B_{2} / 2-B_{1}\right)^{2}}{B_{2}^{2}\left(B_{2}-B_{1}\right)}\left(w-B_{2} / 2\right)+\cdots,
$$

near the point $w=B_{2} / 2$. By assumption, $B_{2}=B_{1} / 2$, so $B_{2} / 2-B_{1} \neq 0$. Since $F\left(z_{2}\right)=B_{2} / 2$ on every sheet of the continuation, we conclude as before that $F$ returns in every continuation to the same function element $f$ at $z_{2}$, and hence, that $F$ is single-valued.

Since $F$ is single-valued, it can have no branch points. Thus the only possible singularities of $F$ are a finite number of poles. This implies that $F$ is a rational function. Consequently, $f$ is also a rational function. By the corollary to Srebro's theorem in $\S 2, f$ has the form (1).

As remarked in $\S 2$, to show that $f$ is a rotation of the Koebe function, it suffices to establish the condition $1-(a / b)^{2}=0$. If $1-(a / b)^{2} \neq 0$, then $f$ has a simple zero at $z=\xi$ for some $|\xi| \geq 1$. Thus near $z=\xi$, the left-hand side of (6) has the form $b(z-\xi)^{-2}+\cdots$ for some constant $b$, which forces $\xi=1 / \bar{\zeta}_{1}$. Similarly, from (7) we obtain $\xi=1 / \bar{\zeta}_{2}$. Hence $\zeta_{1}=\zeta_{2}$, a contradiction; this completes the proof of Theorem 2 .

\section{ACKNOWLEDGMENT}

This paper is part of the author's doctoral thesis written at the University of Michigan under the direction of Peter L. Duren. The author thanks Professor Peter L. Duren for motivation and guidance and for being a constant source of inspiration.

\section{REFERENCES}

1. L. Brickman, Functionals of rational type over the class $S$, Proc. Amer. Math. Soc. 92 (1984), 372-376.

2. P. L. Duren, Univalent functions, Grundlehren Math. Wiss., vol. 259, Springer-Verlag, New York, Berlin, 1983.

3. S. S. Goh, The two-functional conjecture for univalent functions, Ph.D. Thesis, Univ. of Michigan, Ann Arbor, MI, 1992.

4. $ـ$ Functionals of higher derivative type, in progress.

5. K. Pearce, New support points of $S$ and extreme points of $H S$, Proc. Amer. Math. Soc. 81 (1981), 425-428.

6. A. C. Schaeffer and D. C. Spencer, Coefficient regions for schlicht functions, Amer. Math. Soc. Colloq. Publ., vol. 35, Amer. Math. Soc., Providence, RI , 1950.

7. U. Srebro, Is the slit of a rational slit mapping in $S$ straight?, Proc. Amer. Math. Soc. 96 (1986), 65-66.

Department of Mathematics, University of Michigan, ANn ARbor, Michigan 48109

Current address: 6, Lorong Pisang Emas, Singapore 2159, Republic of Singapore 